\title{
Remdesivir, Renal Function and Short-Term Clinical Outcomes in Elderly COVID-19 Pneumonia Patients: A Single-Centre Study
}

\author{
Edoardo Biancalana',* \\ Martina Chiriacò ${ }^{1, *}$ \\ Paolo Sciarrone (D) \\ Alessandro Mengozzi ${ }^{1}$ \\ Sandra Mechelli ${ }^{2}$ \\ Stefano Taddei ${ }^{\text {I }}$ \\ Anna Solini (iD ${ }^{3}$ \\ 'Department of Clinical and \\ Experimental Medicine, University of \\ Pisa, Pisa, Italy; ${ }^{2}$ Section of General \\ Medicine IV AUOP, University of Pisa, \\ Pisa, Italy; ${ }^{3}$ Department of Surgical, \\ Medical, Molecular and Critical Area \\ Pathology, University of Pisa, Pisa, Italy \\ *These authors contributed equally to \\ this work
}

Background: Remdesivir, an antiviral agent able to reduce inflammatory cascade accompanying severe, life-threatening pneumonia, became the first drug approved by the Food and Drug Administration for the treatment of hospitalized patients with coronavirus 2 related severe acute respiratory syndrome (SARS CoV2). As from its previously known clinical indications, the use of remdesivir in the presence of severe renal impairment is contraindicated; however, the impact of remdesivir on renal function in aging patients has not been elucidated.

Subjects and Methods: This retrospective observational study involved 109 individuals consecutively admitted in internal medicine section, Azienda Ospedaliero Universitaria Pisana hospital, in November-December 2020 due to a confirmed diagnosis of SARS $\mathrm{CoV} 2$ and receiving remdesivir according to international inclusion criteria. Biochemical variables at admission were evaluated, together with slopes of estimated glomerular filtration rate (eGFR) built during remdesivir treatment. Participants were followed until discharge or exitus.

Results: Patients were stratified according to age (80 formed the study cohort and 29 served as controls); CKD stage III was present in $46 \%$ of them. No patients showed any sign of deteriorated renal function during remdesivir. Fourteen patients in the elderly cohort deceased; their eGFR at baseline was significantly lower. Recovered patients were characterized by a relevant eGFR gaining during remdesivir treatment.

Conclusion: We show here for the first time as remdesivir does not influence eGFR in a cohort of elderly people hospitalized for SARS CoV2, and that eGFR gain during such treatment is coupled with a better prognosis.

Keywords: remdesivir, SARS CoV2, glomerular filtration rate, aging, chronic kidney disease

\section{Introduction}

Coronavirus Disease 2019 (COVID-19) is an ongoing global pandemic causing significant increases in morbidity and mortality, whose clinical impact is particularly severe for older individuals. ${ }^{1,2}$ Approximately $25 \%$ of deaths due to COVID19 have been registered in people over the age of seventy, whose frequent serious comorbidities act as relevant predisposing factors for a more severe COVID-19 clinical course and consequent death. ${ }^{3,4}$ Such high mortality is prominently due to severe acute respiratory syndrome, able to quickly spread to vulnerable populations such as comorbid elderly individuals with aging-related disorders. The current
Correspondence: Anna Solini Department of Surgical, Medical, Molecular and Critical Area Pathology, University of Pisa, Pisa, I-56I26, Italy

Tel +39-050993482

Fax +39-050553235

Email anna.solini@med.unipi.it 
ongoing pandemic of COVID-19, together with a huge effort to discover new drugs or vaccines able to stop the virus spreading, requires repurposing of existing drugs as safe and effective alternative potentially able to fight the disease. Among these, remdesivir, an ATP-analogue nucleotide with a broad-spectrum of antiviral activity against Ebola, Nipah, respiratory syncytial virus family and a diverse category of coronaviruses including severe acute respiratory syndrome coronavirus 2 (SARS CoV2), ${ }^{5-7}$ is currently utilized. It should be able to inhibit the viral RNA synthesis by a specific mechanism of delayed chain termination; however, its use against COVID-19 has been, so far, characterized by alternate fortune.

The impact of antiviral therapies on the prognosis of SARS CoV2 patients is debated. ${ }^{8}$ Mortality rate, clinical improvement, and discharge have been better among patients receiving remdesivir compared to placebo or standard of care; ${ }^{9}$ however, a recent meta-analysis including five randomized clinical trials resulted in little to no reduction in mortality, and in a small reduction in the proportion of patients receiving mechanical ventilation, although a 5-day course seems to reduce mortality, need for ventilation, and serious adverse events, while increasing the percentage of patients who recovered or clinically improved. ${ }^{10}$ Matter of fact, based on encouraging actions documented in in vitro settings and animal models, ${ }^{11-13}$ along with the promising, although not univocal results of a few randomized clinical trials, ${ }^{14,15}$ it has been authorized for the treatment of SARS CoV2 patients in several countries. ${ }^{16}$ Among the limitations to its use, an estimated glomerular filtration rate (eGFR) $<30 \mathrm{~mL} / \mathrm{min} / 1.73 \mathrm{~m}^{2}$ has been applied in the clinical practice, according to a $74 \%$ renal elimination of remdesivir and its active metabolite, and to the potential accumulation of its vector sulphobutylethere- $\beta$-cyclodextrin (SBE- $\beta-\mathrm{CD}){ }^{17,18}$ However, no information is so far available on the true impact of remdesivir on renal function in aging subjects; the widespread use of this antiviral treatment in the attempt to fight SARS CoV2 should be accompanied by further knowledge on its short-term impact on the kidney, and which could be, if any, the relationship between renal function and COVID-19related clinical outcomes.

Aim of the present study was to evaluate the impact of remdesivir on renal function and clinical outcome in a cohort of elderly individuals hospitalized for SARS $\mathrm{CoV} 2$ and deserving treatment with remdesivir according to the internationally standardized clinical indications for its use.

\section{Subjects and Methods Study Design}

This single-centre, observational, retrospective study was performed in the sections of internal medicine of the University Hospital in Pisa, Italy during the second phase of the current pandemic; patients admitted between November 1st, 2020 and December 20th, 2020 and matching inclusion criteria were included in the analysis. Inclusion criteria were age $>65$ years, CT scanconfirmed diagnosis of interstitial pneumonia SARS $\mathrm{CoV} 2$ related, admission in intermediate intensity-care setting, eligible to treatment with remdesivir according to local infectious disease consortium and international guidelines (positive PCR testing on nasopharyngeal swab; symptom onset $<10$ days; respiratory failure or need of $\mathrm{O}_{2}$ supply and $\mathrm{PaO}_{2} / \mathrm{FiO}_{2}$ ratio $(\mathrm{P} / \mathrm{F})>200 \leq 300$ at blood gas analysis; eGFR $>30 \mathrm{~mL} / \mathrm{min} / 1.73 \mathrm{~m}^{2}$; serum liver enzymes $<5$-fold upper limit). In each subject, medical history, clinical characteristics, presence of chronic comorbidities and ongoing therapies were registered; biochemistry and arterial blood gas analysis at admission were performed on venous and arterial blood samples. In all patients some inflammatory parameters (fibrinogen, ferritin, $\mathrm{C}$ reactive protein (CRP)) were also monitored every two days, together with IL-6, measured by ELISA.

All patients received the same therapeutic protocol, regularly updated according to the scientific literature and described in detail elsewhere, ${ }^{19}$ briefly, dexamethasone $6 \mathrm{mg}$ iv for 10 days and enoxaparin 4000-6000 IU once daily.

Remdesivir was administered applying the five-day EMA protocol: ${ }^{20} 200 \mathrm{mg}$ iv the first day and $100 \mathrm{mg}$ iv in days 2-5. During the 5-day treatment, serum creatinine was measured daily and eGFR slopes were built according to the CKD-EPI formula. Episodes of acute kidney injury (AKI), defined as an increase in serum creatinine by $\geq$ $0.3 \mathrm{mg} / \mathrm{dl}$ within 48 hours (2012 KDIGO guidelines), were also registered. Delta $(\Delta)$ eGFR was calculated as percent of variation of mean eGFR registered at day 4 and 5 of remdesivir treatment, with respect to the value at admission in hospital.

Patients were followed until death or release from the hospital (discharged when matching all the following 
standardized criteria: no fever in the last 48 hours, $\mathrm{satO}_{2}$ $>94 \%$ in ambient air (AA) or $\mathrm{P} / \mathrm{F}>300$ in AA since the last 48 hours, respiratory rate $<22 / \mathrm{min}$ in resting condition, no other clinical acute conditions).

For some analyses, data were compared with those obtained in a group of patients exactly matching the same inclusion criteria, admitted in the same hospital division during the same period and following the same therapeutic protocol (including remdesivir), but aging $<60$ years, who served as controls.

\section{Approvals}

This retrospective, observational study was conducted according to the principles stated in the Declaration of Helsinki, and it conforms to standards currently applied in our country. Ethical approval was obtained from the local institutional review board (Comitato Etico Area Vasta Nord Ovest, Internal Review Board).

\section{Statistics}

Statistical analysis was performed using IBM SPSS Software. Shapiro-Wilk normality test was performed on all continuous variables. Normally distributed variables were presented as mean \pm standard deviation (SD), whereas variables with skewed distribution were presented as median and interquartile range (IR). Categorical data were presented as percentage. Comparative statistics for continuous variables were performed using the independent samples Student's $t$-test or the corresponding nonparametrical Wilcoxon/Kruskal-Wallis Test, as appropriate. Inter-group differences among categorical variables were tested using Pearson's chi-square test with Fisher's exact test, as appropriate. Correlation analyses were conducted using Spearman's rank-order correlation test and regression analysis was conducted where appropriate.

To investigate predictors of the outcome univariate logistic regression was first performed, statistically significant variables $(p<0.05)$ were then included in a multivariable logistic regression analysis to identify independent outcome predictors. All statistical analyses were considered significant with a two-tailed p-value $<0.05$.

\section{Results}

\section{Clinical Characteristics of the Study Cohort}

The study cohort was formed of 80 individuals whose clinical characteristics are shown in Table 1. Mean age was 79 years; $27.5 \%$ of them had type 2 diabetes (T2D) and $46 \%$ had CKD stage III according with the NKF criteria. Half the population was already treated at home with antithrombotic and RAS-active drugs. As expected, all patients showed laboratory signs of a powerful inflammatory state, shown by high fibrinogen and IL-6 levels. Besides age, the main difference with younger individuals was prevalence of some comorbidities (hypertension and atrial fibrillation) and higher HS troponin and brain natriuretic peptide (BNP); as expected mean eGFR at admission was significantly higher in controls. Blood gas analysis parameters at admission did not differ between the two groups.

Stratifying the aging cohort by sex (Suppl Table A), the only different parameters at baseline were serum creatinine at admission, BNP and ferritin, all significantly higher in males; a gender-related difference in ferritin levels has been reported already during SARS CoV2. ${ }^{21}$

As reported in Suppl Table B, patients with T2D did not differ from non-diabetic individuals except for higher random and fasting plasma glucose values and for a higher prevalence of hypertension and heart failure; serum potassium was slightly higher and arterial $\mathrm{pH}$ was lower, even within the normal range.

\section{Outcomes}

Over the permanence in the hospital, 66 patients $(82.5 \%)$ recovered and were discharged (mean staying: 14 days), while 14 deceased. Table 2 shows clinical and biochemical parameters at in-hospital admission; deceased subjects were older, with a significantly lower eGFR at baseline and higher fibrinogen levels; interestingly, $\mathrm{P} / \mathrm{F}$ at admission did not differ between the two groups. Only $4.9 \%$ of the patients participating in the study developed an AKI episode during the observational follow-up.

When we performed such comparison in the subset of twenty-two T2D patients, deceased patients $(n=4)$ were characterized by worse respiratory parameters and, similarly to that observed in the whole study cohort, by a worse baseline renal function at admission (Suppl Table C). Both fasting and random plasma glucose, although not significantly different, tended to be higher in dead patients.

Table 3 shows linear correlations between baseline variables in recovered and deceased aging patients. Inflammatory markers were related one to each other in discharged patients, while in deceased the most significant linear correlations emerged in the subset of T2D patients, with HbA1c relating with inflammatory markers and P/F. eGFR at admission was significantly and inversely correlated with CRP levels in 
Table I Clinical Characteristics of the Study Cohort and Control Group at Hospital Admission

\begin{tabular}{|c|c|c|c|}
\hline & Study Cohort $n=80$ & Controls $n=29$ & p value \\
\hline Age (years) & $79[73,85]$ & $56[50,60]$ & $<0.0001$ \\
\hline Male (n, \%) & $42,52.5$ & $24,82.7$ & 0.0043 \\
\hline Type 2 diabetes (n, \%) & $22,27.5$ & $6,20.7$ & 0.4721 \\
\hline Hypertension (n, \%) & $46,57.5$ & $10,34.5$ & 0.0336 \\
\hline Heart failure (n, \%) & $9 ; 11.2$ & 0 & 0.0593 \\
\hline Atrial fibrillation (n, \%) & $16,20.0$ & 0 & 0.0091 \\
\hline $\operatorname{ASCVD}(\mathrm{n}, \%)$ & 19, 23.7 & $4,13.8$ & 0.2602 \\
\hline ACEi/ARB (n, \%) & $40,50.0$ & $9,31.0$ & 0.0786 \\
\hline Statins (n, \%) & $21,26.2$ & $6,20.7$ & 0.5523 \\
\hline Antithrombotic therapy (n, \%) & $4 I, 5 I .2$ & $5,17.2$ & 0.0015 \\
\hline eGFR $<60 \mathrm{~mL} / \mathrm{min} / 1.73 \mathrm{~m}^{2}(\mathrm{n}, \%)$ & $37,46.2$ & $5,17.2$ & 0.0060 \\
\hline Random plasma glucose $(\mathrm{mg} / \mathrm{dl})$ & $128[1 \mid 3,170]$ & $156[130,194]$ & 0.0137 \\
\hline Serum creatinine $(\mathrm{mg} / \mathrm{dl})$ & $1.0[0.8,1.3]$ & $1.0[0.8,1.2]$ & 0.5606 \\
\hline eGFR $\left(\mathrm{mL} / \mathrm{min} / 1.73 \mathrm{~m}^{2}\right)$ & $60.2 \pm 18.8$ & $81.2 \pm 20.4$ & $<0.0001$ \\
\hline Serum $\mathrm{Na}^{2+}(\mathrm{mEq} / \mathrm{l})$ & $137[135,140]$ & $136[134,139]$ & 0.1088 \\
\hline Serum $\mathrm{K}^{+}(\mathrm{mEq} / \mathrm{l})$ & $4.1 \pm 0.5$ & $4.0 \pm 0.6$ & 0.7600 \\
\hline BUN (mmol/l) & $23[18,32]$ & $18[15,21]$ & 0.0009 \\
\hline CRP (mg/dl) & $6.4[2.3,12.5]$ & $6.1[2.1,10.0]$ & 0.5432 \\
\hline IL-6 (pg/mL) & $19.6[6.8,30.0]$ & $20.7[\mid 3.4,31.6]$ & 0.5595 \\
\hline Ferritin $(\mu g / l)$ & $513[226,816]$ & $731[304,1089]$ & 0.1317 \\
\hline Fibrinogen (mg/dl) & $503 \pm 143$ & $485 \pm 150$ & 0.7154 \\
\hline D-dimer (mg/l) & $0.4[0.3,0.9]$ & $0.3[0.2,0.4]$ & 0.0020 \\
\hline HS Troponine (ng/l) & $20[10,35]$ & $7[7,12]$ & $<0.0001$ \\
\hline $\mathrm{BNP}(\mathrm{pg} / \mathrm{mL})$ & $62[37,187]$ & $18[10,44]$ & $<0.0001$ \\
\hline $\mathrm{pH}$ & $7.46[7.44,7.49]$ & $7.47[7.45,7.50]$ & 0.5394 \\
\hline $\mathrm{PaO}_{2}(\mathrm{mmHg})$ & $61[53,72]$ & $6 \mathrm{I}[54,72]$ & 0.8158 \\
\hline $\mathrm{PaCO}_{2}(\mathrm{mmHg})$ & $34[31,38]$ & $32[30,36]$ & 0.3019 \\
\hline $\mathrm{HCO}^{3-}(\mathrm{mmol} / \mathrm{l})$ & $25.1 \pm 3.2$ & $25.0 \pm 2.5$ & 0.7704 \\
\hline $\mathrm{PaO}_{2} / \mathrm{FiO}_{2}(\mathrm{P} / \mathrm{F})(\mathrm{mmHg})$ & $278[237,316]$ & $283[250,330]$ & 0.4346 \\
\hline
\end{tabular}

Notes: Wilcoxon/Kruskal-Wallis Tests (Rank Sums). Statistically significant differences are in bold.

deceased patients $(\mathrm{r}-0.545, \mathrm{p}<0.05)$ and with HS Troponin levels in discharged patients $(\mathrm{r}-0.368, \mathrm{p}<0.05)$. Regression analysis showed a linear relationship between baseline eGFR and CRP levels in deceased patients ( $\beta-0.256$, CI 95\% $-0.492,-0.02, \mathrm{p}<0.05$ ) (Suppl Figure A).

\section{eGFR During Remdesivir}

We then built the slopes of eGFR during remdesivir treatment, dividing patients according to the clinical outcome. Figure 1 shows as in both subgroups, remdesivir use was associated with a clinically relevant eGFR gaining, which 
Table 2 Clinical and Biochemical Characteristics of the Study Cohort According to Clinical Outcome

\begin{tabular}{|c|c|c|c|}
\hline & Discharged $n=66$ & Deceased $n=14$ & p value \\
\hline Age (years) & $78[72,84]$ & $82[78,90]$ & 0.0218 \\
\hline Male (n, \%) & $32,48.5$ & $10,71.4$ & 0.1184 \\
\hline Type 2 diabetes $(\mathrm{n}, \%)$ & $18,27.2$ & $4,28.5$ & 0.9213 \\
\hline Hypertension (n, \%) & $39,59.0$ & $7,50.0$ & 0.5320 \\
\hline Heart failure (n, \%) & $8,12.1$ & I, 7.1 & 0.5923 \\
\hline Atrial fibrillation (n, \%) & $11,16.6$ & $5,35.7$ & 0.1056 \\
\hline $\operatorname{ASCVD}(\mathrm{n}, \%)$ & $15,22.7$ & $4,28.5$ & 0.6407 \\
\hline ACEi/ARB (n, \%) & $33,50.0$ & $7,50.0$ & 1.000 \\
\hline Statins (n, \%) & $17,25.6$ & $4,28.5$ & 0.8279 \\
\hline Antithrombotic therapy (n, \%) & $32,48.5$ & $9,64.3$ & 0.2827 \\
\hline eGFR $<60 \mathrm{~mL} / \mathrm{min} / \mathrm{l} .73 \mathrm{~m}^{2}(\mathrm{n}, \%)$ & $27,41.0$ & $10,71.4$ & 0.0375 \\
\hline Random plasma glucose $(\mathrm{mg} / \mathrm{dl})$ & $124[107,|7|]$ & $138[126,180]$ & 0.1246 \\
\hline Serum creatinine $(\mathrm{mg} / \mathrm{dl})$ & $1.0[0.8,1.2]$ & I. $2[1.0,1.5]$ & 0.0250 \\
\hline eGFR $\left(\mathrm{mL} / \mathrm{min} / 1.73 \mathrm{~m}^{2}\right)$ & $62.3 \pm 18.2$ & $50.3 \pm 19.3$ & 0.0299 \\
\hline Serum $\mathrm{Na}^{2+}(\mathrm{mEq} / \mathrm{l})$ & $138[135,140]$ & $137[134,143]$ & 0.9554 \\
\hline Serum $\mathrm{K}^{+}(\mathrm{mEq} / \mathrm{l})$ & $4.0 \pm 0.5$ & $4.2 \pm 0.5$ & 0.4809 \\
\hline $\mathrm{BUN}(\mathrm{mmol} / \mathrm{l})$ & $22[18,27]$ & $31[18,39]$ & 0.0886 \\
\hline CRP (mg/dl) & $5.5[2.1,12.3]$ & $9.2[5.0,17.0]$ & 0.0530 \\
\hline IL-6 (pg/mL) & $19.6[6.8,29.6]$ & $18.6[7.4,32.5]$ & 0.8098 \\
\hline Ferritin $(\mu g / l)$ & $439[210,816]$ & $690[335,1188]$ & 0.2594 \\
\hline Fibrinogen (mg/dl) & $487 \pm 142$ & $581 \pm 126$ & 0.0249 \\
\hline D-dimer (mg/l) & $0.5[0.3,0.9]$ & $0.4[0.3,1.3]$ & 0.6239 \\
\hline HS Troponine (ng/l) & $25.1 \pm 23.1$ & $31.2 \pm 17.8$ & 0.4027 \\
\hline BNP $(\mathrm{pg} / \mathrm{mL})$ & $173 \pm 276$ & $149 \pm 137$ & $0.767 \mid$ \\
\hline $\mathrm{pH}$ & $7.46 \pm 0.05$ & $7.46 \pm 0.03$ & 0.9832 \\
\hline $\mathrm{PaO}_{2}(\mathrm{mmHg})$ & $69.7 \pm 22.4$ & $67.5 \pm 37.7$ & 0.2274 \\
\hline $\mathrm{PaCO}_{2}(\mathrm{mmHg})$ & $34.6 \pm 5.0$ & $33.2 \pm 7.4$ & 0.3194 \\
\hline $\mathrm{HCO}^{3-}(\mathrm{mmol} / \mathrm{l})$ & $25.4 \pm 3.0$ & $23.4 \pm 3.4$ & 0.0400 \\
\hline $\mathrm{PaO}_{2} / \mathrm{FiO}_{2}(\mathrm{P} / \mathrm{F})(\mathrm{mmHg})$ & $28 I[240,329]$ & $252[201,290]$ & 0.0623 \\
\hline
\end{tabular}

Notes: Wilcoxon/Kruskal-Wallis Tests (Rank Sums). Statistically significant differences are in bold.

was almost two-fold higher that those observed in deceased patients (mean $\triangle \mathrm{eGFR}$ day 4 -day $1:+20.9 \%$ vs $+11.4 \%$ ); however, while patients encountering a favourable outcome were characterized by a rather stable eGFR during remdesivir administration, deceased subjects displayed a more fluctuating trend of the slope, with a larger variability of absolute eGFR values (Figure 1).

Suppl Figure B shows eGFR slopes in the subset of aging patients with CKD at baseline: the two subgroups started from a similar eGFR (44.6 \pm 9.2 in survivors and 
Table 3 Matrix of Univariate Correlations Between Variables of Interest in Elderly Discharged (A) and Deceased (B) Patients

\begin{tabular}{|c|c|c|c|c|c|c|c|c|}
\hline (A) & Age & RPG & HbAlc & eGFR & CRP & IL-6 & Fibr & HS TnT \\
\hline P/F & -0.028 & -0.375 & 0.001 & 0.113 & -0.333 & 0.175 & $-0.14 \mid$ & 0.118 \\
\hline HS TnT & 0.429 & -0.001 & -0.384 & -0.368 & 0.164 & 0.334 & 0.233 & - \\
\hline Fibrinogen & 0.032 & 0.372 & -0.019 & -0.128 & 0.600 & 0.426 & - & - \\
\hline IL-6 & -0.025 & 0.125 & 0.243 & -0.231 & 0.306 & - & - & - \\
\hline CRP & 0.104 & 0.333 & -0.153 & -0.156 & - & - & - & - \\
\hline eGFR & -0.339 & -0.248 & -0.103 & - & - & - & - & - \\
\hline HbAlc & $-0.47 I$ & 0.549 & - & - & - & - & - & - \\
\hline RPG & 0.205 & - & - & - & - & - & - & - \\
\hline (B) & Age & RPG & HbAlc & eGFR & CRP & IL-6 & Fibr & HS TnT \\
\hline P/F & -0.063 & -0.149 & -0.379 & -0.008 & -0.036 & 0.482 & -0.347 & 0.038 \\
\hline HS TnT & 0.475 & 0.084 & 0.974 & -0.234 & 0.081 & 0.079 & 0.211 & - \\
\hline Fibrinogen & 0.042 & 0.308 & -0.683 & -0.255 & 0.348 & -0.223 & - & - \\
\hline IL-6 & 0.067 & -0.452 & -1.000 & -0.252 & 0.205 & - & - & - \\
\hline CRP & 0.146 & 0.201 & 0.696 & -0.545 & - & - & - & - \\
\hline eGFR & -0.517 & -0.269 & -0.869 & - & - & - & - & - \\
\hline HbAlc & -0.666 & 0.990 & - & - & - & - & - & - \\
\hline RPG & -0.188 & - & - & - & - & - & - & - \\
\hline
\end{tabular}

Notes: HbAlc available only in T2D individuals $(n=22) . \rho$ values with $p<0.05$ are shown in bold.

41.0 $\pm 9.7 \mathrm{~mL} / \mathrm{min} / 1.73 \mathrm{~m}^{2}$ ) in deceased, but these ones, differently from survivors, did not show any relevant eGFR improvement during remdesivir treatment (mean $\triangle \mathrm{eGFR}:+30 \pm 41 \%$ vs $+9 \pm 40 \%, \mathrm{p}=0.245)$.

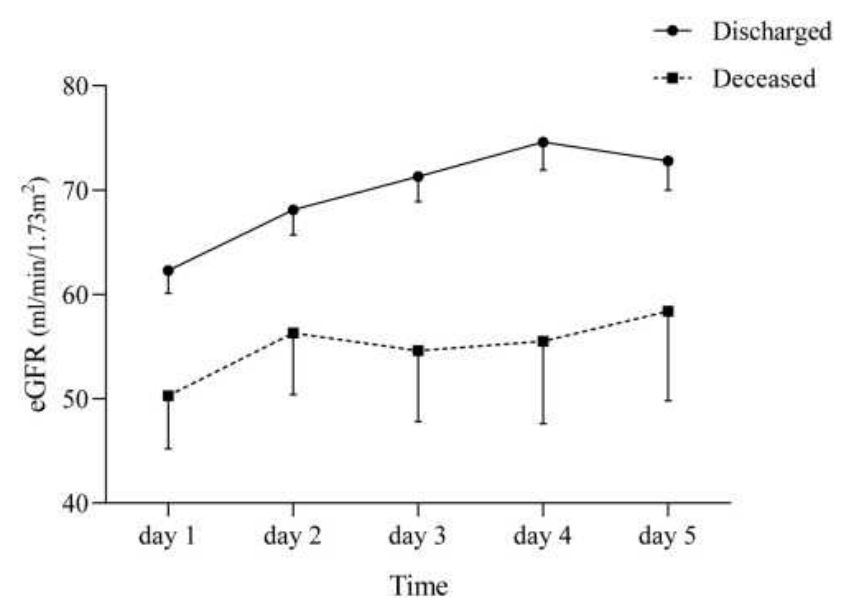

Figure I Glomerular function of the study cohort of elderly patients in relation to clinical outcome. Data are shown as mean \pm SE.

\section{Predictors of Outcome}

By univariate analysis, age, fibrinogen, CRP and baseline eGFR were predictors of mortality (Table 4); however, when grouped together in a multivariable model, none such variables remained as significant independent predictors of mortality, even after testing for interactions with age and sex. This was true also in the subset of T2D patients, in which none of the explored variables was able to predict the main outcome (data not shown).

In the group of younger patients serving as controls, three individuals out of twenty-nine (10.3\%) deceased. eGFR at baseline was $81.0 \pm 7.4$ and $87.8 \pm 6.6 \mathrm{~mL} / \mathrm{min} /$ $1.73 \mathrm{~m}^{2}$ in discharged and deceased, respectively $(\mathrm{p}=\mathrm{ns})$. Suppl Figure C Upper Panel shows eGFR slopes in such group according to the prognosis: discharged were clearly characterized by a relevant eGFR increase during remdesivir treatment $\left(+18.5 \pm 15.2 \mathrm{~mL} / \mathrm{min} / 1.73 \mathrm{~m}^{2}\right)$. Suppl Figure C Lower Panel compares eGFR slopes of discharged individuals: in both groups, although starting 
Table 4 Univariate and Multivariate Analysis in the Whole Cohort of Elderly Patients $(n=80)$

\begin{tabular}{|c|c|c|c|c|c|c|}
\hline \multirow[t]{2}{*}{ Variable } & \multicolumn{3}{|c|}{ Univariate } & \multicolumn{3}{|c|}{ Multivariate } \\
\hline & OR & Cl & $\mathbf{p}$ & OR & Cl & $\mathbf{p}$ \\
\hline Age & 1.102 & $1.015-1.196$ & 0.021 & & & \\
\hline Sex & 2.656 & $0.756-9.327$ & 0.127 & & & \\
\hline Hospital stay & 0.939 & $0.864-1.019$ & 0.131 & & & \\
\hline Hypertension & 0.692 & $0.218-2.202$ & 0.533 & & & \\
\hline Heart failure & 0.558 & $0.064-4.856$ & 0.597 & & & \\
\hline ASCVD & 1.360 & $0.373-4.963$ & 0.642 & & & \\
\hline Atrial fibrillation & 2.778 & $0.780-9.896$ & 0.115 & & & \\
\hline Type 2 diabetes & 1.067 & $0.297-3.836$ & 0.921 & & & \\
\hline CKD $\geq$ stage 3 & 1.870 & $0.584-5.985$ & 0.292 & & & \\
\hline $\mathrm{PaO}_{2} / \mathrm{FiO}_{2}(\mathrm{P} / \mathrm{F})$ & 0.990 & $0.980-1.000$ & 0.061 & & & \\
\hline BNP & 1.000 & $0.997-1.002$ & 0.763 & & & \\
\hline HS Troponine & 1.011 & $0.985-1.037$ & 0.403 & & & \\
\hline Ferritin & 1.001 & $0.999-1.002$ & 0.323 & & & \\
\hline CRP & 1.099 & $1.013-1.193$ & 0.024 & 1.038 & $0.938-1.149$ & $0.47 \mid$ \\
\hline IL-6 & 1.002 & $0.944-1.063$ & 0.960 & & & \\
\hline Fibrinogen & 1.005 & $1.000-1.010$ & 0.030 & 1.003 & $0.998-1.009$ & 0.230 \\
\hline D-dimer & 1.045 & $0.824-1.326$ & 0.715 & & & \\
\hline Serum $\mathrm{Na}^{2+}$ & 0.967 & $0.918-1.019$ & 0.212 & & & \\
\hline Serum $\mathrm{K}^{+}$ & 1.924 & $0.7 \mid I-5.207$ & 0.198 & & & \\
\hline Random plasma glucose & 1.003 & $0.994-1.012$ & 0.473 & & & \\
\hline eGFR at admission & 0.963 & $0.929-0.998$ & 0.036 & $0.97 \mid$ & $0.934-1.009$ & 0.139 \\
\hline$\Delta$ eGFR & 10.266 & $0.019-3.707$ & 0.324 & & & \\
\hline
\end{tabular}

Note: Statistically significant differences are in bold.

from a significantly different eGFR, regained renal function was evident, with a similar trend over time.

\section{Discussion}

During the first months of the pandemic, the promising inhibition properties of remdesivir toward SARS-CoV-2 have been identified, and, based on encouraging results obtained in a few placebo-controlled human studies showing a better recovery of COVID-19 positive hospitalized patients, ${ }^{22,23}$ these observations have been rapidly translated into a clinical use of this molecule in fighting the most serious symptoms and signs of SARS CoV2 in adults; however, the behavior of renal function during such treatment in elderly people has not been detailed.

This single-center observational study shows, for the first time, as remdesivir: i) does not worsen renal function in elderly individuals with and without CKD; ii) intriguingly, several patients show a relevant eGFR improvement during remdesivir administration; such trend seems to be coupled with an increased probability of recovering from SARS CoV2 and a better prognosis.

Impaired renal function is a proven major determinant of all-cause and cardiovascular mortality in the general population and in patients carrying several acute and chronic comorbidities ${ }^{24-26}$ and marks an unfavorable prognosis in bacterial and viral infections. ${ }^{27,28}$ In our cohort of elderly individuals hospitalized for SARS CoV2, common indicators of renal function at the admission and, to some extent, recovery of kidney function during hospitalization, influenced their prognosis, even in the presence of a disease whose main target organ is, apparently, the lung. This confirms that the kidney is primarily involved in SARS CoV2 infection, ${ }^{29,30}$ likely via the high affinity of SARS CoV2 virus for ACE2, ${ }^{31}$ and reinforces the importance of preserved renal function as a major prognostic determinant in patients hospitalized for SARS CoV2 pneumonia.

In deceased elderly subjects, a reduced renal function at admission was inversely related with CRP, the most 
widely used indicator of inflammation, able to predict disease severity in COVID-19 patients. $^{32}$ This confirms a possible role the systemic inflammatory condition as determinant of impaired renal function ${ }^{33-35}$ or, possibly, reflects the effects on the kidney of a more severe viral infection, determining a multiorgan failure.

The eGFR slopes during remdesivir administration deserve to be commented on. The first observation regards the renal safety of remdesivir, which did not induce any eGFR loss, and this was true irrespective of the presence of CKD stage III. Even more, both survivors and patients who died during hospitalization quite rapidly increased their eGFR while receiving remdesivir, although the phenomenon was more evident in the former group. This could be simply ascribed to their better eGFR at baseline, already described as determinant of renal function improvement after antiretroviral therapies; ${ }^{36}$ alternatively, this ameliorated kidney function might reflect a better response to therapies, with an improved function of several organs and tissues.

The subgroup of CKD patients (Suppl Figure B) displays a quite peculiar difference in eGFR trend over remdesivir treatment: constant and progressive increase over time in discharged vs small oscillations characterizing the slope of deceased ones. This might suggest that the improvement in glomerular function during recovery, together with eGFR at admission, influence the clinical outcome in severely ill SARS CoV2 patients with chronically impaired renal function. Of note, individuals with type 2 diabetes did not differ from non-diabetic ones in terms of eGFR slope or performance of prognostic markers (data not shown), again suggesting a main role played by the kidney, rather than other comorbidities, in influencing clinical course, and even prognosis, of these individuals.

Despite the described association between SARS CoV2 and acute renal damage, ${ }^{37}$ and although the study cohort included a considerable proportion of patients with CKD, only a very small percentage developed AKI during the first days of hospitalization, concomitant to remdesivir treatment. This aspect deserves particular attention. Before the pandemic, the clinical experience with remdesivir, developed to fight RNA viruses with global pandemic potential, including SARS and MERS coronaviruses and in particular Ebola, was rather limited. We show here, by a real-life observation in elderly, frail people, as remdesivir is safe on the kidney and its use is not coupled with any sign of acute deterioration of renal function; even more, as already pointed out, some patients encountered an eGFR rise during the treatment. This finding might reflect a possible nephroprotective action of remdesivir that has not yet been described; we might only speculate that remdesivir might promptly counteract SARS CoV2-mediated direct, acute damage in the proximal tubular epithelium and of podocytes detachment from the glomerular basement membrane, together with the role played by the attenuation of systemic inflammation. Another possibility, that unfortunately we have been unable to test in patients participating in this survey, could be a decrease in ACE2 activity. ${ }^{38,39}$

Matter of fact, in such cohort of patients with severe pneumonia and respiratory failure, no variable among those routinely checked was able to independently predict their short-term prognosis, and this was also true for the $\mathrm{PaO}_{2} / \mathrm{FiO}_{2}$ ratio, an established indicator of respiratory failure severity in people carrying SARS CoV2, underlying the complexity of the disease, with systemic involvement of multiple tissues and organs.

The main strength of this study resides in the detailed and repeated eGFR monitoring performed during the treatment, with no missing data due to the strict daily monitoring of several biochemical variables, the nature of the study cohort, with casual high prevalence of CKD, standardized conditions of the observation with strict rules for receiving the drug. We should however acknowledge several limitations, including the retrospective nature of the study, the relatively small number of subjects, the existence of pre-defined clinical indications to remdesivir treatment that cannot allow to generalize the possible clinical meaning of this observation.

\section{Conclusions}

Taken together these observations reassure on the safe use of remdesivir with respect to renal function in SARS CoV2 elderly patients, pointing out as the evaluation of eGFR slope during the antiviral treatment could integrate respiratory parameters and inflammation markers, providing a better prognostic phenotype of such frail patients.

\section{Abbreviations}

AA, ambient air; AKI, acute kidney injury; BNP, brain natriuretic peptide; $\mathrm{CKD}$, chronic kidney disease; COVID19, Coronavirus Disease 2019; CRP, C reactive protein; eGFR, estimated glomerular filtration rate; HS, Troponin: high sensitivity troponin; IL-6, interleukin 6; P/F, $\mathrm{PaO}_{2} / \mathrm{FiO}_{2}$ ratio; RAS, renin angiotensin system; SARS CoV2, severe acute respiratory syndrome coronavirus 2; T2D, type 2 diabetes. 


\section{Data Sharing Statement}

The datasets used and/or analysed during the current study are not publicly available due to privacy concerns. They are available from the corresponding author on reasonable request.

\section{Ethics Approval and Consent to Participate}

Ethical approval was obtained from the local institutional review board (Comitato Etico Area Vasta Nord Ovest, Internal Review Board). All patients provided consent for the anonymous use of data from their clinical records for scientific purposes.

\section{Acknowledgments}

We thank Dr Simone Leonetti for his excellent supervision of statistics. Edoardo Biancalana and Martina Chiriacò are co-first authors in this study.

\section{Author Contributions}

All authors made substantial contributions to conception and design, acquisition of data, or analysis and interpretation of data; took part in drafting the article or revising it critically for important intellectual content; agreed to submit to the current journal; gave final approval of the version to be published; and agree to be accountable for all aspects of the work.

\section{Disclosure}

The authors report no conflicts of interest in this work.

\section{References}

1. Pijls BG, Jolani S, Atherley A, et al. Demographic risk factors for COVID-19 infection, severity, ICU admission and death: a meta-analysis of 59 studies. BMJ Open. 2021;11(1):e044640. doi:10.1136/bmjopen-2020-044640

2. Shiels MS, Almeida JS, García-Closas M, Albert PS, Freedman ND, Berrington de González A. Impact of population growth and aging on estimates of excess U.S. deaths during the COVID-19 pandemic, march to august 2020. Ann Intern Med. 2020;M20-7385.

3. Smorenberg A, Peters EJ, van Daele P, Nossent EJ, Muller M. How does SARS-CoV-2 targets the elderly patients? A review on potential mechanisms increasing disease severity. Eur $J$ Intern Med. 2021;83:1-5. doi:10.1016/j.ejim.2020.11.024

4. Yifan C, Jun P. Understanding the clinical features of Coronavirus disease 2019 from the perspective of aging: a systematic review and meta-analysis. Front Endocrinol (Lausanne). 2020;11:557333. doi:10.3389/fendo.2020.557333

5. Malin JJ, Suárez I, Priesner V, Fätkenheuer G, Rybniker J. Remdesivir against COVID-19 and other viral diseases. Clin Microbiol Rev. 2020;34(1):e00162-20. doi:10.1128/CMR.00162-20

6. Lo MK, Feldmann F, Gary JM, et al. Remdesivir (GS-5734) protects African green monkeys from Nipah virus challenge. Sci Transl Med. 2019;11(494):eaau9242. doi:10.1126/scitranslmed.aau9242
7. Hong YN, Xu J, Sasa GBK, Zhou KX, Ding XF. Remdesivir as a broad-spectrum antiviral drug against COVID-19. Eur Rev Med Pharmacol Sci. 2021;25(1):541-548. doi:10.26355/eurrev_ 202101_24426

8. Perveen RA, Nasir M, Talha KA, Selina F, Islam MA. Systematic review on current antiviral therapy in COVID-19 pandemic. Med J Malaysia. 2020;75(6):710-716.

9. Shrestha DB, Budhathoki P, Syed NI, Rawal E, Raut S, Khadka S. Remdesivir: a potential game-changer or just a myth? A systematic review and meta-analysis. Life Sci. 2021;264:118663. doi:10.1016/j. lfs. 2020.118663

10. Kaka AS, MacDonald R, Greer N, et al. Major update: remdesivir for adults with COVID-19: a living systematic review and meta-analysis for the American College of Physicians Practice Points. Ann Intern Med. 2021;M20-8148.

11. Pruijssers AJ, George AS, Schäfer A, et al. Remdesivir inhibits SARS-CoV-2 in human lung cells and chimeric SARS-CoV expressing the SARS-CoV-2 RNA polymerase in mice. Cell Rep. 2020;32 (3):107940. doi:10.1016/j.celrep.2020.107940

12. Simonis A, Theobald SJ, Fätkenheuer G, Rybniker J, Malin JJ. A comparative analysis of remdesivir and other repurposed antivirals against SARS-CoV-2. EMBO Mol Med. 2021;13(1):e13105. doi:10.15252/emmm.202013105

13. Zhang Y, Tang LV. Overview of targets and potential drugs of SARS-CoV-2 according to the viral replication. J Proteome Res. 2021;20(1):49-59. doi:10.1021/acs.jproteome.0c00526

14. ACTT-1 Study Group Members; Beigel JH, Tomashek KM, Dodd LE, et al. Remdesivir for the treatment of Covid-19 - final report. NEngl JMed. 2020;383(19):1813-1826. doi:10.1056/NEJMoa2007764.

15. Wang Y, Zhang D, Du G, et al. Remdesivir in adults with severe COVID-19: a randomised, double-blind, placebo-controlled, multicentre trial. Lancet. 2020;395(10238):1694. doi:10.1016/S01406736(20)31022-9

16. Nasir M, Perveen RA, Murshed M, Nazneen R, Talha KA. Survival and biomarkers of COVID-19 patients treated with remdesivir and favipiravir in ICU during the peak of pandemic: a single center study in Bangladesh. J Pharm Res Int. 2021;32(45):14-22. doi:10.9734/ jpri/2020/v32i4531088

17. Hoover RK, Alcorn H, Lawrence L, et al. Clinical pharmacokinetics of sulfobutylether- $\beta$-cyclodextrin in patients with varying degrees of renal impairment. J Clin Pharmacol. 2018;58(6):814-822. doi: $10.1002 /$ jcph. 1077

18. Kiser TH, Fish DN, Aquilante CL, et al. Evaluation of sulfobutylether- $\beta$-cyclodextrin (SBECD) accumulation and voriconazole pharmacokinetics in critically ill patients undergoing continuous renal replacement therapy. Crit Care. 2015;19(1):32. doi:10.1186/s13054015-0753-8

19. Pisa COVID-19 Study Group; Falcone M, Tiseo G, Barbieri G, et al. Role of low-molecular-weight heparin in hospitalized patients with severe acute respiratory syndrome Coronavirus 2 pneumonia: a prospective observational study. Open Forum Infect Dis. 2020;7 (12):ofaa563. doi:10.1093/ofid/ofaa563.

20. European Medicines Agency. Summary on compassionate use. Remdesivir. Gilead. European Medicines Agency, Amsterdam, The Netherlands; 2020. Available from: https://www.ema.europa.eu/en/ documents/other/summary-compassionate-use-remdesivir-gilead_en. pdf. Accessed May 18, 2021.

21. Yoshida Y, Gillet SA, Brown MI, et al. Clinical characteristics and outcomes in women and men hospitalized for coronavirus disease 2019 in New Orleans. Biol Sex Differ. 2021;12(1):20. doi:10.1186/ s13293-021-00359-2

22. GS-US-540-5774 Investigators; Spinner CD, Gottlieb RL, Criner GJ, et al. Effect of remdesivir vs standard care on clinical status at 11 days in patients with moderate COVID-19: a randomized clinical trial. JAMA. 2020;324(11):1048-1057. doi:10.1001/jama.2020.16349. 
23. GS-US-540-5773 Investigators; Goldman JD, Lye DCB, Hui DS, et al. Remdesivir for 5 or 10 days in patients with severe Covid-19. $N$ Engl J Med. 2020;383(19):1827-1837. doi:10.1056/ NEJMoa2015301.

24. Coresh J, Turin TC, Matsushita K, et al. Decline in estimated glomerular filtration rate and subsequent risk of end-stage renal disease and mortality. JAMA. 2014;311(24):2518-2531. doi:10.1001/ jama.2014.6634

25. Chronic Kidney Disease Prognosis Consortium; Fox CS, Matsushita K, Woodward M, et al. Associations of kidney disease measures with mortality and end-stage renal disease in individuals with and without diabetes: a meta-analysis. Lancet. 2013;381 (9864):374. doi:10.1016/S0140-6736(13)60273-1.

26. CKD Prognosis Consortium; Matsushita K, Coresh J, Sang Y, et al. Estimated glomerular filtration rate and albuminuria for prediction of cardiovascular outcomes: a collaborative meta-analysis of individual participant data. Lancet Diabetes Endocrinol. 2015;3(7):514-525. doi:10.1016/S2213-8587(15)00040-6.

27. Du X, Xu X, Yao J, et al. Predictors of mortality in patients infected with carbapenem-resistant Acinetobacter baumannii: a systematic review and meta-analysis. Am J Infect Control. 2019;47 (9):1140-1145. doi:10.1016/j.ajic.2019.03.003

28. Fabrizi F, Donato FM, Messa P. Hepatitis C and its metabolic complications in kidney disease. Ann Hepatol. 2017;16(6):851-861. doi: $10.5604 / 01.3001 .0010 .5275$

29. Farkash EA, Wilson AM, Jentzen JM. Ultrastructural evidence for direct renal infection with SARS-CoV-2. J Am Soc Nephrol. 2020;31 (8):1683-1687. doi:10.1681/ASN.2020040432

30. Su H, Yang M, Wan C, et al. Renal histopathological analysis of 26 postmortem findings of patients with COVID-19 in China. Kidney Int. 2020;98(1):219-227. doi:10.1016/j.kint.2020.04.003

31. Perico L, Benigni A, Remuzzi G. Should COVID-19 concern nephrologists? Why and to what extent? The emerging impasse of angiotensin blockade. Nephron. 2020;144(5):213-221. doi:10.1159/ 000507305
32. Tjendra Y, Al Mana AF, Espejo AP, et al. Predicting disease severity and outcome in COVID-19 patients: a review of multiple biomarkers. Arch Pathol Lab Med. 2020;144(12):1465-1474. doi:10.5858/ arpa.2020-0471-SA

33. Fan F, Jia J, Li J, Huo Y, Zhang Y. White blood cell count predicts the odds of kidney function decline in a Chinese community-based population. BMC Nephrol. 2017;18(1):190. doi:10.1186/s12882017-0608-4

34. Arnold LW, Hoy WE, Wang Z. The association between C-reactive protein levels and the risk for chronic kidney disease hospitalizations in adults of a remote Indigenous Australian community a prospective cohort study. Nephrology (Carlton). 2017;22 (9):699-705. doi:10.1111/nep.12841

35. Gao J, Wang A, Li X, et al. The cumulative exposure to high-sensitivity C-reactive protein predicts the risk of chronic kidney diseases. Kidney Blood Press Res. 2020;45(1):84-94. doi:10.1159/ 000504251

36. Mackanga JR, Alène C, Doumingou N, et al. The baseline glomerular filtration rate, predictive of six-year survival in sub-Saharan African patients on antiretroviral therapy for HIV: cohort study. Nephrol Ther. 2019;15(4):220-225. doi:10.1016/j.nephro.2019.02.009

37. Liu YF, Zhang Z, Pan XL, et al. The chronic kidney disease and acute kidney injury involvement in COVID-19 pandemic: a systematic review and meta-analysis. PLoS One. 2021;16(1):e0244779. doi:10.1371/journal.pone.0244779

38. Rodríguez-Romo R, Benítez K, Barrera-Chimal J, et al. AT1 receptor antagonism before ischemia prevents the transition of acute kidney injury to chronic kidney disease. Kidney Int. 2016;89(2):363-373. doi:10.1038/ki.2015.320

39. Sharma RK, Li J, Krishnan S, Richards EM, Raizada MK, Mohandas R. Angiotensin-converting enzyme 2 and COVID-19 in cardiorenal diseases. Clin Sci (Lond). 2021;135(1):1-17. doi:10.1042/ CS20200482
Clinical Interventions in Aging

\section{Publish your work in this journal}

Clinical Interventions in Aging is an international, peer-reviewed journal focusing on evidence-based reports on the value or lack thereof of treatments intended to prevent or delay the onset of maladaptive correlates of aging in human beings. This journal is indexed on PubMed Central, MedLine, CAS, Scopus and the Elsevier

\section{Dovepress}

Bibliographic databases. The manuscript management system is completely online and includes a very quick and fair peer-review system, which is all easy to use. Visit http://www.dovepress.com/ testimonials.php to read real quotes from published authors. 